\title{
In situ delivery of passive immunity by lactobacilli producing single-chain antibodies
}

\author{
Carina Krüger ${ }^{1}$, Yanzhong Huํ, Qiang Pan ${ }^{1}$, Harold Marcotte ${ }^{1}$, Anna Hultberg ${ }^{1}$, Dipu Delwar ${ }^{1}$, \\ Philip J. van Dalen², Peter H. Pouwels ${ }^{2,3}$, Rob J. Leer ${ }^{2,3}$, Charles G. Kelly ${ }^{4}$, Craig van Dollenweerd ${ }^{4}$, \\ Julian K. Ma ${ }^{4}$, and Lennart Hammarström ${ }^{1}$
}

\begin{abstract}
Lactobacilli have previously been used to deliver vaccine components for active immunization in vivo. Vectors encoding a single-chain Fv (scFv) antibody fragment, which recognizes the streptococcal antigen I/II (SAI/II) adhesion molecule of Streptococcus mutans, were constructed and expressed in Lactobacillus zeae (American Type Culture Collection (ATCC) 393). The scFv antibody fragments secreted into the supernatant or expressed on the surface of the bacteria showed binding activity against SAI/II in enzyme-linked immunosorbent assay (ELISA), and surface scFv-expressing lactobacilli agglutinated SAI/II-expressing S. mutans in vitro without affecting the corresponding SAI/II knockout strain. Lactobacilli expressing the scFv fragment fused to an E-tag were visualized by scanning electron microscopy (SEM) using beads coated with a monoclonal anti-E-tag antibody, and they bound directly to beads coated with SAI/II. After administration of scFv-expressing bacteria to a rat model of dental caries development, S. mutans bacteria counts and caries scores were markedly reduced. As lactobacilli are generally regarded as safe (GRAS) microorganisms, this approach may be of considerable commercial interest for in vivo immunotherapy.
\end{abstract}

Streptococcus mutans is the major pathogen involved in caries development through production of lactic acid ${ }^{1-4}$. Two virulence factors participate in the colonization process. The first, surface antigen SAI/II (also known as B, IF, P1, SR, MSL-1, and $\mathrm{Pac}^{5}$ ), is an adhesin that binds to salivary pellicles. The second is a series of surface glucosyltransferases (GTFs) that catalyze the synthesis of glucan ${ }^{6}$, contributing to the later stages of the adherence process.

Local passive immunotherapy can prevent colonization by S. mutans, and oral administration of hyperimmune bovine $\operatorname{IgG}^{7}$ or chicken IgY ${ }^{8}$ markedly lowers the caries score in S. mutans-infected rats and reduces the bacterial load in humans ${ }^{9,10}$. Similar therapeutic results have been obtained by applying a mouse monoclonal IgG1 antibody, Guy's 13, directed against the SAI/II adhesion molecule on S. mutans, to the teeth of both primates ${ }^{11,12}$ and humans ${ }^{13,14}$. Guy's 13 antibodies, with the $\mathrm{CH} 3$ domain replaced by the $\mathrm{CH} 2$ and $\mathrm{CH} 3$ domains of a mouse $\alpha$-chain, have recently been developed and produced in plants ${ }^{15}$. These antibodies effectively prevent colonization of the teeth by S. mutans in human volunteers ${ }^{16}$, suggesting a new approach in prevention of oral infections using genetically engineered immunoglobulins.

$\mathrm{ScFv}$ fragments have been produced in numerous systems ${ }^{17-20}$ and used for the treatment of infectious disease ${ }^{21-23}$. As the antibody fragments have a very short half-life in vivo, new methods must be developed to increase their longevity before they can be therapeutically applied at mucosal sites.

Lactobacilli are Gram-positive bacteria that have been used in food fermentation and preservation for centuries ${ }^{24}$, and some strains are normal constituents of the human intestinal microflora ${ }^{25}$. Several authors have also suggested that fermented products positively affect the course of certain infectious diseases ${ }^{26-29}$. Selected strains of Lactobacillus casei and Lactobacillus plantarum have also been shown to exert strong adjuvant effects on the mucosal and the systemic immune respons $\mathrm{e}^{30-32}$. Furthermore, lactobacilli have been suggested as carriers for oral vaccines ${ }^{33}$ and expression vectors have previously been constructed for surface expression or secretion of various antigens $s^{32,34-37}$.

We have expressed Guy's $13 \mathrm{scFv}$ both on the surface of L. zeae ATCC 393 (previously named L. casei ATCC 393) and as a secreted protein. The recombinant $\mathrm{scFv}$ recognized SAI/II and the transformed lactobacilli acted therapeutically in experimental animals, both decreasing the number of $S$. mutans bacteria and reducing the development of caries. Our results thus suggest an approach for in situ delivery of passive immunity at mucosal sites.

\section{Results}

Construction of scFv-expressing L. zeae. The scFv-encoding gene derived from the variable regions ( $\mathrm{VH}$ and $\mathrm{VL}$ ) of Guy's 13 (IgG1) ${ }^{11}$, with addition of an E-tag-encoding sequence, was inserted into the vector pTUAT $^{35}$. A short or a long anchor sequence of the proteinase $\mathrm{P}$-encoding gene PrtP was introduced, generating pTUAT-A (Fig. 1A). The scFv-containing or the scFv-anchor-containing DNA fragments were then cloned into the Escherichia coli/Lactobacillus shuttle vector pLP402 (Fig. 1B). A terminator from the lactate dehydrogenase gene ( $\mathrm{Tldh}$ ) was present between the C-terminal region of amylase and the $\mathrm{N}$-terminal end of the scFv to suppress the expression of the downstream sequences in E. coli. The Tldh was removed by NotI digestion of

${ }^{1}$ Center for Oral Biology, Karolinska Institutet at NOVUM and Division of Clinical Immunology, Karolinska Institutet at Huddinge Hospital, SE-141 86 Huddinge, Sweden. ${ }^{2}$ TNO Prevention and Health, Division of Immunology and Infectious Disease, PO Box 2215, 2301 CE Leiden, The Netherlands. ${ }^{3}$ TNO Voeding,

Department of Applied Microbiology and Gene Technology, Postbox 370, 3600 AJ, Zeist, The Netherlands. ${ }^{4}$ Department of Oral Medicine and Pathology, King's College London, Guy's Hospital, London Bridge, London, UK. *Corresponding author (lennart.hammarstrom@biosci.ki.se). 
A

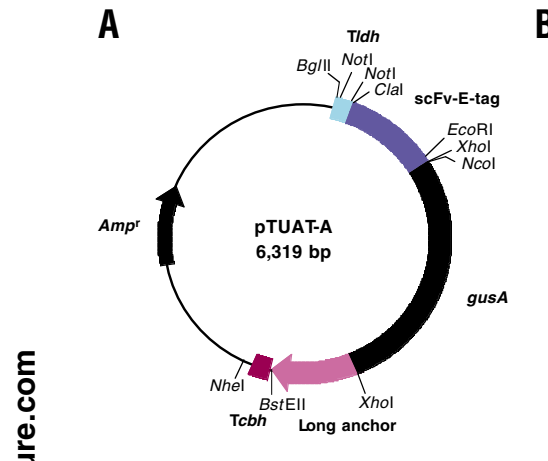

C

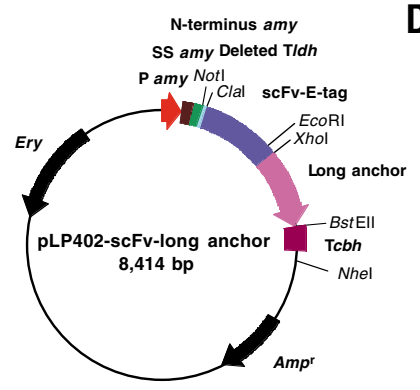

B

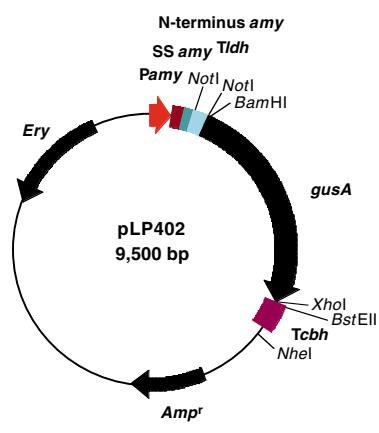

Figure 1. Map of Lactobacillus expression vectors. (A) Intermediate vector pTUAT-A, in which the Sc-sense-etag-antisense PCR amplicon was cloned between the Clal and Ncol sites of pTUAT ${ }^{35}$. (B) Lactobacillus expression vector pLP402. (C) pLP402-scFv-long anchor vector mediating surfaceanchored expression of scFv by fusion to the last 244 amino acids of $L$. zeae proteinase P. (D) pLP402-scFv-secreted vector mediating secretion of scFv in the medium. Pamy, Promoter sequence of the $\alpha$-amylase gene of L. amylovorus; SS amy, secretion signal of the $\alpha$-amylase gene of L. amylovorus ( 36 amino acids); N-terminus amy, $\mathrm{N}$-terminus (26 amino acids) of the $\alpha$-amylase gene of $L$. amylovorus; $T / d h$, transcription terminator of the lactate dehydrogenase gene of $L$. zeae; gusA, gene marker encoding $\beta$-glucuronidase; long anchor, anchor sequence from the proteinase $\mathrm{P}$ gene of $L$. zeae (244 amino acids); $T c b h$, transcription terminator sequence of the conjugated bile acid hydrolase gene of $L$. plantarum $80 ; A m p^{r}$, ampicillinresistance gene; Ery, erythromycin-resistance gene; deleted T/dh, remaining sequence after deletion of $\mathrm{T} / \mathrm{dh}$.

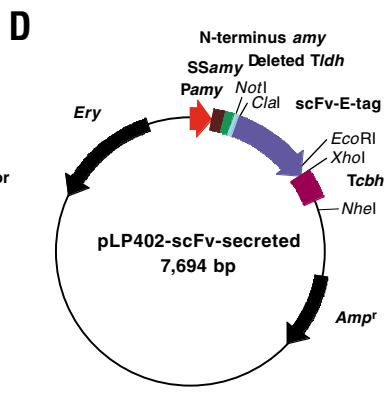

the plasmid, and after ligation the resulting vectors were introduced into L. zeae ATCC 393. Three different constructs were made: (i) the pLP402-scFv-short anchor vector, mediating cell-surface expression by fusion to the last 117 amino acids of the proteinase P of L. zeae ATCC 393; (ii) the pLP402-scFv-long anchor vector mediating cell-surface expression by fusion to the last 244 amino acids of the proteinase $P$ protein (Fig. 1C); and (iii) pLP402-scFv, mediating secretion of scFv into the medium (Fig. 1D). The theoretical molecular masses of the expressed proteins from each vector were $47 \mathrm{kDa}, 60 \mathrm{kDa}$, and $35 \mathrm{kDa}$, respectively.

Expression of scFv. ScFv expression was analyzed by immunoblotting of L. zeae-transformed strains using monoclonal anti-E-tag antibodies. The cell extract of $L$. zeae transformed with the pLP402scFv-long anchor-encoding vector yielded a $60 \mathrm{kDa}$ protein (Fig. 2A), and the culture supernatant of $L$. zeae transformed with the pLP402S-scFv vector contained a protein of $\sim 40 \mathrm{kDa}$ (Fig. 2B).

Surface expression of $\mathrm{scFv}$ in recombinant $L$. zeae strains transformed with the pLP402-scFv-short anchor and pLP402-scFv-long anchor was also analyzed by flow cytometry using an anti-E-tag antibody. The pLP402-scFv-long anchor-transformed lactobacilli showed a strong positive signal when stained by the anti-E-tag antibody, whereas the lactobacilli transformed with the pLP402-scFv-short anchor vector showed a less intense signal (Fig. 2C). This confirms the surface location of $\mathrm{scFv}$ in the two recombinant $L$. zeae strains containing the scFv-anchor fusion constructs.

Biological activity of the scFv. The biological activity of the recombinant $s c F v$ was analyzed by both ELISA and agglutination assays. ScFvs from homogenates of pLP402-scFv-short anchor- and pLP402scFv-long anchor-transformed lactobacilli and culture supernatant of pLP402-scFv transformants were tested in ELISA (Table 1). The scFvs from all recombinant strains bound to SAI/II coated to microtiter plates (Table 1), although the pLP402-scFv-short anchor construct bound less well than the pLP402-scFv-long anchor construct.

The streptococci or lactobacilli used in the agglutination assay showed no self-agglutination. Streptococcus mutans NG8 (wild type), expressing the SAI/II antigen, was rapidly co-agglutinated with $L$. zeae transformed with the pLP402-scFv-long anchor construct, whereas no co-agglutination occurred when a mutant $S$. mutans 834 strain (SAI/II knockout) was used (Table 2). Mixing of S. mutans NG8 and L. zeae transformed with the pLP402-scFv-short anchor construct resulted in weak agglutination only (Table 2). Furthermore, the time required for visible aggregation was more than double that of L. zeae transformed with the pLP402-scFv-long anchor construct (73 and $33 \mathrm{~s}$, respectively, mean of five experiments).

The transformed lactobacilli were also incubated with magnetic polystyrene beads (Dynal M-450) and coated with anti-E-tag antibodies; a scanning electron microscope was used to visualize the binding. The binding of pLP402-scFv-long anchor-transformed bacteria (Fig. 3A), but not the negative control, L. zeae pLP402 (Fig. 3B), to the anti-E-tag-coated beads was determined by SEM. Furthermore, pLP402-scFv-long anchor-transformed lactobacilli bound directly to SAI/IIcoated magnetic polystyrene beads (Dynal M-450 Epoxy; Fig. 3C, D).

Therapeutic effect in vivo. To be therapeutically effective, antibody-producing lactobacilli must persist in the oral cavity for a prolonged period of time. Desalivated rats were therefore orally inoculated with transformed lactobacilli (pLP402, pLP402-scFv, pLP402-scFv-short anchor, and pLP402-scFv-long anchor), and the presence of bacteria was measured as the presence of erythromycinresistant colonies containing the $\mathrm{scFv}$ insert. When rats were inoculated with the transformed lactobacilli every second day for a period of two weeks, the bacteria persisted for the duration of the experiment (three weeks; data not shown). Thus, bacteria could still be detected one week after the final inoculation. Lactobacilli were eluted

the table.

${ }^{b}$ Absorbance at $405 \mathrm{~nm}$ after $60 \mathrm{~min}$ incubation with substrate.

cTotal protein concentrations were $6.0,1.3$, and $1.5 \mathrm{mg} / \mathrm{ml}$, respectively.

dSupernatant of a mouse hybridoma producing the Guy's 13 antibody (concentration $10 \mu \mathrm{g} / \mathrm{ml}$ ). 
from a cotton swab from the oral cavity and nontransformed bacteria colonized more effectively (approximately 10,000 colonies per swab) than transformants (approximately 1,000 colonies per swab). However, there was no major difference in colonizing capacity between the bacteria harboring the different constructs.

Rats treated with L. zeae transformed with the pLP402-scFv-long anchor construct had a significantly lower number of $S$. mutans NG8 in the oral cavity as compared with rats infected with lactobacilli transformed with pLP402 and the S. mutans control (Table 3). Significantly fewer animals in the pLP402-scFv-long anchor group developed dental caries lesions as compared with both the pLP402-containing lactobacilli and the S. mutans control groups (Table 3). The mean number of caries lesions was also significantly different between the pLP402scFv-long anchor-expressing Lactobacillus group and the $S$. mutans control group (25.6 and 42.3, respectively; $P<0.05$ (Student's $t$-test)).

\section{Discussion}

Most infectious agents enter by the mucosal route, and locally produced IgM and IgA antibodies may readily prevent disease. Oral administration of human, bovine, and chicken immunoglobulins has also effected prophylactic and therapeutic responses against a variety of gastrointestinal infections ${ }^{38-42}$.

The majority of antibody preparations used to date have been polyclonal in origin, but a monoclonal antibody will suffice for protection against many infectious diseases or mitigation of an ongoing infection. The latter may be produced by hybridoma technology, in transgenic plants, or by microorganisms such as E. coli and yeast. However, these modes of production are fraught with disadvantages and all require purification of the antibodies before administration.

Vaccine antigens and cytokines have been expressed in vivo in the gastrointestinal tract by transformed lactobacilli ${ }^{34}$ and lacto$\operatorname{cocci}^{43}$. The benefit of using this system for delivery of antibodies or antibody fragments was exploited in our experiments, in which antibodies against a mucosal pathogen, $S$. mutans, were produced by lactobacilli. Theoretically, bacteria colonizing different anatomical sites within the oro-gastrointestinal tract might be selectively used and transformed with vectors encoding antibodies against a variety of pathogens. The long persistence of the transformed lactobacilli in the oral cavity and their therapeutic efficacy suggests that this may be a viable approach for future therapy either prophylactically or therapeutically. In vivo production of antibody fragments locally in the intestine circumvents the practical problem of degradation of orally administered antibodies in the stomach.

Table 2. Co-agglutination of pLP402-scFv-long anchor-transformed lactobacilli with wild-type (NG8) and SAI/II-defective (834) S. mutans

\begin{tabular}{|c|c|c|}
\hline L. zeae transformants & S. mutans & Agglutination \\
\hline- & NG8 & - \\
\hline- & Mutant (834) (SAI/II ko) & - \\
\hline pLP402 & - & - \\
\hline pLP402 & NG8 & - \\
\hline pLP402 & Mutant (834) (SAI/II ko) & - \\
\hline pLP402-scFv-short anchora & - & - \\
\hline pLP402-scFv-short anchor ${ }^{a}$ & NG8 & + \\
\hline pLP402-scFv-short anchor ${ }^{a}$ & Mutant (834) (SAI/II ko) & - \\
\hline pLP402-scFv-long anchora & - & - \\
\hline pLP402-scFv-long anchor ${ }^{a}$ & NG8 & +++ \\
\hline pLP402-scFv-long anchor ${ }^{a}$ & Mutant (834) (SAI/II ko) & - \\
\hline
\end{tabular}

${ }^{a}$ Expression of scFv was induced by growth in LCM medium containing mannitol as described. Agglutination was assayed arbitrarily, where + corresponds to agglutination of $20 \%$ of the bateria and +++ corresponds to $90 \%$ agglutination. ko, Knockout.
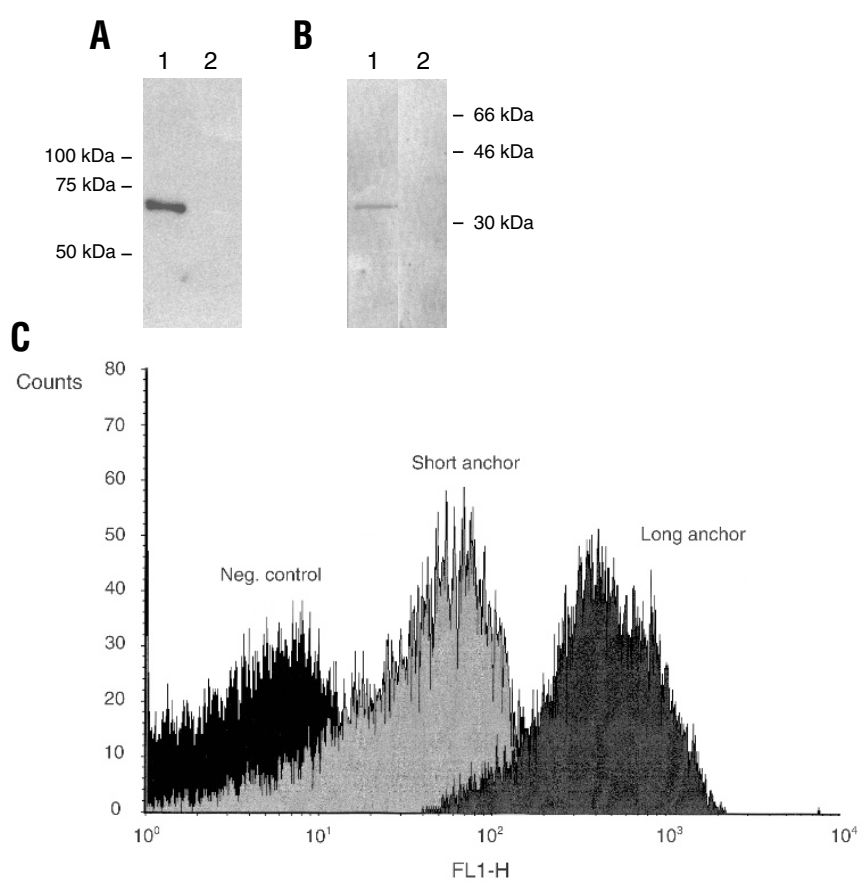

Figure 2. Detection of the scFv expressed by L. zeae by immunoblotting and fluorescence-activated cell sorting (FACS). (A) The homogenate of lactobacilli transformed by the pLP402-scFv-long anchor (lane 1) and pLP402 vectors (lane 2). (B) Concentrated supernatant of Lactobacillus transformant pLP402-scFv-secreted (lane 1) and pLP402 vectors (lane 2). (C) FACS analysis of lactobacilli transformed with the pLP402, pLP402-scFv-short anchor, or pLP402-scFv-long anchor vectors using a primary mouse E-tag monoclonal antibody and fluorescein isothiocyanate-conjugated goat anti-mouse immunoglobulin.

The vectors used in our experiments contain an inducible amylase promoter, and thus the transformed bacteria, after in vitro induction, would be expected to produce scFv fragments only for a limited time in vivo, hence the need for repeated administration. As it may be more efficient to use a constitutive promoter, such vectors are currently being constructed.

Our present experiments utilized the Guy's 13 antibody as a parent antibody for the construction of the scFv. This is one of the most wellcharacterized, therapeutically effective mouse monoclonal antibodies described to date ${ }^{11-16}$. The suggested mechanism of action of the Guy's 13 antibody involves agglutination of the $S$. mutans bacteria, as Fab fragments, in contrast to $\mathrm{Fab}_{2}$ fragments, do not prevent recolonization ${ }^{14}$. The secreted scFv, also functionally monovalent, would therefore be expected to be therapeutically inferior. Lactobacilli expressing monovalent $\mathrm{scFv}$ on their surfaces, however, would be anticipated to work effectively as "biological beads" because of the multivalent binding, resulting in high avidity. In our experiments, this may have promoted agglutination and subsequent clearance of bacteria. The bound S. mutans bacteria might also be effectively killed by the high local concentration of bactericidal and bacteriostatic proteins produced by the lactobacilli. Although model $\mathrm{scFv}$ fragments have been expressed by Gram-positive bacteria such as staphylococci (anti-IgE) ${ }^{44}$ and streptococci (anti-candida) $)^{45}$, this is the first report to show that antibody fragments can be produced by lactobacilli, thus providing a basis for future application of in vivo delivery of passive immunity in humans.

\section{Experimental protocol}

Construction of the single-chain antibody gene. The single-chain antibody gene fragment encoding the Guy's 13 monoclonal antibody (scFv, $711 \mathrm{bp}$ ) was amplified from $\mathrm{CDNA}$ and cloned into the pCANTAB $5 \mathrm{E}$ phage vector 

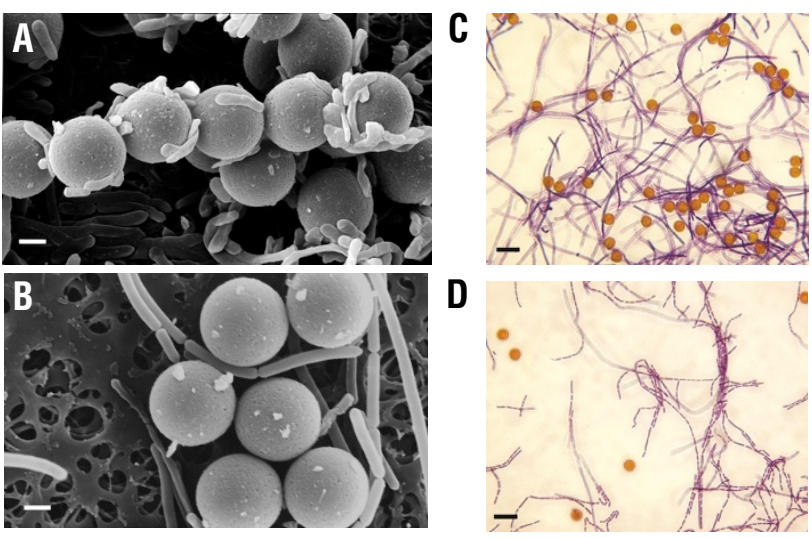

Figure 3. Adherence of transformed lactobacilli to magnetic beads coated with E-tag antibodies or SAI/II. (A) L. zeae pLP402-scFv-long anchor transformants adhere to the beads coated with E-tag antibodies (magnification $\times 5,000$; bar, $2 \mu \mathrm{m}$ ). (B) L. zeae pLP402 transformants (with empty vector) do not adhere to the beads coated with anti-E-tag antibodies (magnification $\times 5,000$; bar, $2 \mu \mathrm{m}$ ). (C) L. zeae pLP402scFv-long anchor transformants adhere to the beads coated with SAI/II (magnification $\times 1,000$; bar, $11 \mu \mathrm{m}$ ). (D) L. zeae pLP402 transformants (empty vector) do not bind to the beads coated with SAI/II and most of them are washed away during the preparation procedure (magnification $\times 1,000$; bar, $11 \mu \mathrm{m})$.

(Amersham Biosciences, Uppsala, Sweden) at the SfiI and NotI restriction enzyme sites, generating pCANT-scFv. The $3^{\prime}$ terminus of the scFv-encoding gene was fused with an E-tag-encoding gene to allow serological detection. The NotI restriction enzyme site between the scFv- and the E-tag-encoding genes was replaced by an EcoRI restriction site, as NotI digestion was later used to remove the Tldh terminator present downstream of the $\alpha$-amylase gene-derived expression signals in the pLP402 vector (Fig. 1B).

The $\mathrm{scFv}$ was amplified from the pCANT-scFv vector using primers Sc-sense (5'-CCCATCGATGCCCAGGTGAAACTG- $\left.3^{\prime}\right)$ and Sc-antisense (5'-CGGAATTCCGCC CGTTTTATTTCCA-3') and cloned into pBluescript II SK (+) (Stratagene, La Jolla, CA) at the ClaI and EcoRI sites, generating the pBS vector. The E-tag fragment was generated from the same template as above (pCANT-scFv) using the primers tag-sense (5'-CGGAATTCGGTGCGCCGGTGC CGTAT- $3^{\prime}$ ) and pcant-antisense (5'-TCCCCGCGGTACCAGCGCCAAAGACATAAGG - $3^{\prime}$ ) and was inserted at the EcoRI and SacII sites of pBS, generating the pBSE construct.

Construction of the Lactobacillus expression vectors. A series of scFv expression vectors were constructed containing the promoter and secretion signal sequence of the regulated $\alpha$-amylase gene of L. amylovorus as described elsewhere ${ }^{35,46}$. ScFv was produced in L. zeae ATCC 393, either anchored on the cell surface or secreted in a free form in the medium. The scFv-E-tag fragment from the pBSE construct was amplified with two sets of primers: Sc-sense and etag-antisense A (5'-CATGCCATGGCCTCGAGTGCGGCACGCGGT-3'), and Sc-sense and etag-antisense B (5'-CATGCCATGGCCTCGAGCTATGCGGCACGCGGT-3'), respectively; both of the antisense primers are located downstream of the E-tag and contain $\mathrm{XhoI}$ and $\mathrm{NcoI}$ sites. A stop codon (underlined) was introduced in the etag-antisense B primer for the construction of the

\begin{tabular}{|c|c|c|c|c|c|}
\hline Lactobacilli & $n$ & S. mutans $s^{a}$ & $P$ value $^{\text {b }}$ & Dental caries $^{c}$ & $P$ value ${ }^{\mathrm{d}}$ \\
\hline - & 6 & 2,900 & - & $4(67 \%)$ & - \\
\hline pLP402 & 6 & 2,350 & NS & $4(67 \%)$ & NS \\
\hline $\begin{array}{l}\text { pLP402-scFv- } \\
\text { long anchor }\end{array}$ & 7 & 75 & $<0.05$ & $1(14 \%)$ & $<0.05$ \\
\hline
\end{tabular}

aSummary of two screenings of all individual animals during the final week of the therapeutic experiment. Results are given as median numbers of $S$. mutans colonies. ${ }^{b}$ Mann-Whitney test. NS, Not significant.

'Number of animals with dental (slight and moderate) caries at term of the experiment. ${ }^{d} \chi^{2}$ test. secretion vector. The PCR products from both amplification reactions were subcloned into an E. coli vector, pTUAT, between the $\mathrm{ClaI}$ and $\mathrm{NcoI}$ sites, generating pTUAT-A (Fig. 1A) and pTUAT-B, respectively. For the construction of the expression vector encoding an anchored product, the $\mathrm{Tldh}$, the $\mathrm{scFv}$, the anchor region, and the Tcbh were excised from pTUAT-A with BglII and NheI and cloned into pLP402 between the BamHI and NheI restriction sites. The reporter gene gusA was deleted from the vector using digestion with $X$ hoI followed by ligation with T4 ligase and introduction into E. coli. Subsequently, the Tldh was deleted from the construct by digestion with NotI followed by ligation, generating the pLP402-ScFv-short anchor vector. A construct with an elongated anchor was also made by replacing the existing anchor in pTUAT by the last $732 \mathrm{bp}$ of the coding region of the PrtP gene of L. zeae ATCC 393 (Fig. 1C). The long anchor is approximately twice the size (244 amino acids) of the short anchor (117 amino acids). The fragment was amplified using primers p6612 (5'-GCCGGAATTCCTCGAG AAGAAGACTTCGCTGCTTAACC-3') and p7343 (5'-GCCGGATCCGGTCACCC TATTCTTCACGTTGTTTCCG-3') and cloned into pTUAT between the $X h o \mathrm{I}$ and BstEII sites, generating pTUAT-1. The procedure for the generation of the pLP402-scFv-long anchor vector was subsequently the same as that described for the construction of the pLP402-scFv-short anchor vector. For the construction of the secretion vector, the scFv-E-tag fragment was excised from pTUAT-B using BglII and $\mathrm{XhoI}$ and inserted between the $B a m \mathrm{HI}$ and XhoI restriction sites of pLP402. The Tldh was thereafter deleted as described elsewhere, generating the pLP402-scFv-secreted vector (Fig. 1D).

Escherichia coli JM109 (Stratagene) was used as the host strain for the construction of shuttle vectors and these were introduced by heat shock. Lactobacilli were transfected by electroporation (Gene Pulser II, Bio-Rad Laboratories, Hercules, CA; $25 \mu \mathrm{F}, 100 \Omega$ and $6,250 \mathrm{~V} / \mathrm{cm})^{34}(1 \mu \mathrm{g}$ of plasmid DNA/100 $\mu \mathrm{l}$ of lactobacilli).

Immunoblot analysis of Lactobacillus transformants. To induce expression, cells were grown in Lactobacillus carrying medium (LCM) supplemented with $0.5 \%$ (wt/vol) mannitol for derepression of the amy promoter. Cells were disrupted in $400 \mu \mathrm{l}$ PBS by sonication $(4 \times 10 \mathrm{~s}$ pulses $)$ and debris was removed by centrifugation. Proteins were separated by SDS-PAGE gel (12\% wt/vol) and transferred by electroblotting to $0.2 \mu \mathrm{m}$ nitrocellulose membranes. The culture supernatant was concentrated 50× using ultrafiltration (Amicon, Beverly, MA) and then processed as above. Immunoblots were developed using mouse monoclonal anti-E-tag antibody (Amersham Biosciences) and horseradish peroxidase (HRP)-conjugated goat anti-mouse IgG Fc antibody (1:1,000; Dako, Glostrup, Denmark). Enhanced chemiluminescence (ECL) buffer (Amersham Biosciences) was used to detect the immunoblotting signal, and the rainbow protein marker (Amersham Biosciences) was used as a molecular mass standard.

ELISA and flow cytometry. Purified SAI/II antigen $(3 \mu \mathrm{g} / \mathrm{ml})$ was coated in 96-well ELISA plates (Nunc, Roskilde, Denmark). Supernatant or cell homogenates containing the scFv was added and anti-E-tag antibody and alkaline phosphatase-conjugated rabbit anti-mouse immunoglobulin (Dako) were subsequently added. Flow cytometry was carried out according to standard protocols and the samples were analyzed using a FACSCalibur (Becton Dickinson, Stockholm, Sweden).

Agglutination assay. S. mutans NG8 (SAI/II expressing) and S. mutans 834 (SAI/II knockout) strains ${ }^{47}$ were cultivated to stationary phase in brain-heart infusion ( Difco, Sparks, MD) and harvested by centrifugation. Streptococcus mutans (30 $\mu \mathrm{l}$ suspension) was placed on a glass slide $(76 \times 26 \mathrm{~mm}$, MenzelGläser, Braunschweig, Germany), after which $30 \mu \mathrm{l}$ of L. zeae transformants pLP402, pLP402-scFv-short anchor, or pLP402-scFv-long anchor was added. The slide was then rotated vertically by hand and agglutination was arbitrarily scored to determine when visible aggregation first occurred, and then scored again $2 \mathrm{~min}$ after the start of the assay.

Electron microscopy. Magnetic polystyrene beads (Dynal M-450 Epoxy; Dynal Biotech, Oslo, Norway) precoated with rat anti-mouse IgG were incubated with the mouse monoclonal anti-E-tag antibody, diluted 1:200, and subsequently incubated with $L$. zeae bacteria transformed with either the pLP402-scFv-long anchor or the pLP402 vector. Beads were analyzed by SEM (JEOL JSM-820, Tokyo, Japan) at $15 \mathrm{kV}$.

Magnetic polystyrene beads (Dynal M-450 Epoxy; Dynal Biotech) were also coated with SAI/II at a concentration of $4.5 \mu \mathrm{g} / 10^{7}$ beads, and $6 \times 10^{5}$ beads were mixed with $L$. zeae transformants pLP402 or pLP402-scFv-long anchor and loaded on a glass slide. The slides were rotated for $10 \mathrm{~s}$, Gramstained, and observed under microscope (Olympus, Hamburg, Germany). 
In vivo colonization study. Rat experiments were all approved by the animal ethics committee of southern Stockholm. Female SPF Sprague-Dawley rats were desalivated through surgery at 21 days of age. This procedure facilitates rapid development of caries ${ }^{48}$. During two successive days, groups of animals were infected with either nontransformed bacteria or lactobacilli transformed with pLP402, pLP402-scFv, pLP402-scFv-short anchor, or pLP402-scFv-long anchor vectors. Rats were inoculated by swabbing the oral cavity after dipping the swab in a bacterial suspension containing $5 \times 10^{10}$ colony-forming units $/ \mathrm{ml}$. Thereafter, the groups were infected every second day until day 14 . The rats were screened for growth of lactobacilli every third day until day 14 and thereafter every second day until termination at 20 days after desalivation, using oral swabs plated on Rogosa (Merck, Darmstadt, Germany) agar plates, with erythromycin $(3 \mu \mathrm{g} / \mathrm{ml})$ or without. PCR was used for detection of the inserted scFv gene fragment in erythromycin-resistant colonies.

Caries study. Nineteen SPF Sprague-Dawley rats, 21 days old, were desalivated through surgery ${ }^{48}$. During two successive days, groups of animals were swabbed with sterile water, L. zeae pLP402, or L. zeae pLP402-
scFv-long anchor-transformed bacteria. At 24 and 25 days of age, all groups were infected with $S$. mutans bacteria. Fresh cultures of the Lactobacillus transformants were subsequently given every day in the drinking water to the relevant groups, and all animals were screened for numbers of $S$. mutans and L. zeae every third day by oral swabbing. The animals were all fed a diet of ground food supplemented with $56 \%$ sucrose. At 42 days of age the animals were killed and scored blindly for dental caries by the method of Keyes ${ }^{49}$.

\section{Acknowledgments \\ This work was supported by the Swedish Medical Research Council and by the Foundation for Preventive Caries Research (Patentmedelsfonden).}

\section{Competing interests statement}

The authors declare that they have no competing financial interests.

Received 5 December 2001; accepted 20 March 2002
1. Krasse, B., Edwardsson, S., Svensson, I. \& Trell, L. Implantation of caries-inducing streptococci in the human oral cavity. Arch. Oral Biol. 12, 231-236 (1967).

2. Krasse, B. \& Carlsson, J. Various types of streptococci and experimental caries in hamsters. Arch. Oral Biol. 15, 25-32 (1970).

3. Lehner, T., Challacombe, S.J. \& Caldwell, J. Immunologic basis for vaccination against dental caries in rhesus monkeys. J. Dent. Res. 55, C166-C180 (1976).

4. Loesche, W.J., Rowan, J., Straffon, L.H. \& Loos, P.J. Association of Streptococcus mutants with human dental decay. Infect. Immun. 11, 1252-1260 (1975).

5. Russell, M.W. Immunization against dental caries. Curr. Opin. Dent. 2, 72-80 (1992).

6. Loesche, W.J. Role of Streptococcus mutans in human dental decay. Microbiol. Rev. 50, 353-380 (1986).

7. Michalek, S.M. et al. Protection of gnotobiotic rats against dental caries by passive immunization with bovine milk antibodies to Streptococcus mutans. Infect Immun. 55, 2341-2347 (1987).

8. Hamada, S. et al. Oral passive immunization against dental caries in rats by use of hen egg yolk antibodies specific for cell-associated glucosyltransferase of Streptococcus mutans. Infect. Immun. 59, 4161-4167 (1991).

9. Filler, S.J., Gregory, R.L., Michalek, S.M., Katz, J. \& McGhee, J.R. Effect of immune bovine milk on Streptococcus mutans in human dental plaque. Arch. Oral Biol. 36, 41-47 (1991).

10. Hatta, H. et al. Passive immunization against dental plaque formation in humans: effect of a mouth rinse containing egg yolk antibodies (IgY) specific to Streptococcus mutans. Caries Res. 31, 268-274 (1997)

11. Lehner, T., Caldwell, J. \& Smith, R. Local passive immunization by monoclonal antibodies against streptococcal antigen $\mathrm{I} / \mathrm{I}$ in the prevention of dental caries. Infect. Immun. 50, 796-799 (1985).

12. Lehner, T., Ma, J.K. \& Kelly, C.G. A mechanism of passive immunization with monoclonal antibodies to a $185,000 \mathrm{M}(\mathrm{r})$ streptococcal antigen. Adv. Exp. Med. Biol. 327, 151-163 (1992)

13. Ma, J.K., Smith, R. \& Lehner, T. Use of monoclonal antibodies in local passive immunization to prevent colonization of human teeth by Streptococcus mutans. Infect. Immun. 55, 1274-1278 (1987).

14. Ma, J.K., Hunjan, M., Smith, R., Kelly, C. \& Lehner, T. An investigation into the mechanism of protection by local passive immunization with monoclonal antibodies against Streptococcus mutans. Infect. Immun. 58, 3407-3414 (1990).

15. Ma, J.K. et al. Generation and assembly of secretory antibodies in plants. Science 268, 716-719 (1995)

16. Ma, J.K. et al. Characterization of a recombinant plant monoclonal secretory antibody and preventive immunotherapy in humans. Nat. Med. 4, 601-606 (1998).

17. Little, M., Kipriyanov, S.M., Le Gall, F. \& Moldenhauer, G. Of mice and men: hybridoma and recombinant antibodies. Immunol. Today 21, 364-370 (2000).

18. Lorenzen, N. et al. Immunoprophylaxis in fish by injection of mouse antibody genes. Nat. Biotechnol. 18, 1177-1180 (2000).

19. Giddings, G., Allison, G., Brooks, D. \& Carter, A. Transgenic plants as factories for biopharmaceuticals. Nat. Biotechnol. 18, 1151-1155 (2000)

20. Mottershead, D.G., Alfthan, K., Ojala, K., Takkinen, K. \& Oker-Blom, C. Baculoviral display of functional ScFv and synthetic IgG-binding domains. Biochem. Biophys. Res. Commun. 275, 84-90 (2000).

21. Lamarre, A., Yu, M.W., Chagnon, F. \& Talbot, P.J. A recombinant single chain antibody neutralizes coronavirus infectivity but only slightly delays lethal infection of mice. Eur. Immunol. 27, 3447-3455 (1997).

22. Magliani, W. et al. Therapeutic potential of antiidiotypic single chain antibodies with yeast killer toxin activity. Nat. Biotechnol. 15, 155-158 (1997).

23. Matthews, R., Hodgetts, S. \& Burnie, J. Preliminary assessment of a human recombinant antibody fragment to hsp90 in murine invasive candidiasis. J. Infect. Dis. 171, 1668-1671 (1995).

24. de Vos, W.M. Gene expression systems for lactic acid bacteria. Curr. Opin. Microbiol. 2, 289-295 (1999).

25. Ahrne, S. et al. The normal Lactobacillus flora of healthy human rectal and oral mucosa. J. Appl. Microbiol. 85, 88-94 (1998).

26. Shornikova, A.V., Casas, I.A., Isolauri, E., Mykkanen, H. \& Vesikari, T. Lactobacillus reuteri as a therapeutic agent in acute diarrhea in young children. J. Pediatr. Gastroenterol. Nutr. 24, 399-404 (1997).
27. Gionchetti, P., Rizzello, F., Venturi, A. \& Campieri, M. Probiotics in infective diarrhea and inflammatory bowel diseases. J. Gastroenterol. Hepatol. 15, 489-493 (2000).

28. Guandalini, S. et al. Lactobacillus GG administered in oral rehydration solution to children with acute diarrhea: a multicenter European trial. J. Pediatr. Gastroenterol. Nutr. 30, 54-60 (2000).

29. Vanderhoof, J.A. et al. Lactobacillus GG in the prevention of antibiotic-associated diarrhea in children. J. Pediatr. 135, 564-568 (1999).

30. Perdigon, G., de Macias, M.E., Alvarez, S., Oliver, G. \& de Ruiz Holgado, A.P. Systemic augmentation of the immune response in mice by feeding fermented milks with Lactobacillus casei and Lactobacillus acidophilus. Immunology 63, 17-23 (1988)

31. Isolauri, E., Joensuu, J., Suomalainen, H., Luomala, M. \& Vesikari, T. Improved immunogenicity of oral $\mathrm{D} \times \mathrm{RRV}$ reassortant rotavirus vaccine by Lactobacillus casei GG. Vaccine 13, 310-312 (1995).

32. Pouwels, P.H., Leer, R.J. \& Boersma, W.J. The potential of Lactobacillus as a carrier for oral immunization: development and preliminary characterization of vector systems for targeted delivery of antigens. J. Biotechnol. 44, 183-192 (1996).

33. Pouwels, P.H. et al. Lactic acid bacteria as antigen delivery vehicles for oral immunization purposes. Int. J. Food Microbiol. 41, 155-167 (1998).

34. Shaw, D.M. et al. Engineering the microflora to vaccinate the mucosa: serum immunoglobulin $G$ responses and activated draining cervical lymph nodes following mucosal application of tetanus toxin fragment C-expressing lactobacilli. Immunology 100, 510-518 (2000).

35. Pouwels, P.H. et al. Lactobacilli as vehicles for targeting antigens to mucosal tissues by surface exposition of foreign antigens. Methods Enzymol. 336, 369-389 (2001).

36. Piard, J.C., Jimenez-Diaz, R., Fischetti, V.A., Ehrlich, S.D. \& Gruss, A. The M6 protein of Streptococcus pyogenes and its potential as a tool to anchor biologically active molecules at the surface of lactic acid bacteria. Adv. Exp. Med. Biol. 418, 545-550 (1997)

37. Savijoki, K., Kahala, M. \& Palva, A. High level heterologous protein production in Lactococcus and Lactobacillus using a new secretion system based on the Lactobacillus brevis S-layer signals. Gene 186, 255-262 (1997).

38. Eibl, M.M., Wolf, H.M., Furnkranz, H. \& Rosenkranz, A. Prevention of necrotizing enterocolitis in low-birth-weight infants by IgA-IgG feeding. N. Engl. J. Med. 319, 1-7 (1988).

39. Tjellström, B., Stenhammar, L., Eriksson, S. \& Magnusson, K.E. Oral immunoglobulin A supplement in treatment of Clostridium difficile enteritis. Lancet 341, 701-702 (1993)

40. Hammarström, V., Smith, C.I. \& Hammarström, L. Oral immunoglobulin treatment in Campylobacter jejuni enteritis. Lancet 341, 1036 (1993)

41. Casswall, T.H. et al. Oral IgA-IgG treatment of chronic non-specific diarrhea in infants and children. Acta. Paediatr. 85, 1126-1128 (1996).

42. Weiner, C. et al. Passive immunity against human pathogens using bovine antibodies. Clin. Exp. Immunol. 116, 193-205 (1999).

43. Steidler, L. et al. Treatment of murine colitis by Lactococcus lactis secreting interleukin-10. Science 289, 1352-1355 (2000).

44. Gunneriusson, E., Samuelson, P., Uhlen, M., Nygren, P.A. \& Stahl, S. Surface display of a functional single-chain Fv antibody on staphylococci. J. Bacteriol. 178, 1341-1346 (1996)

45. Beninati, C. et al. Therapy of mucosal candidiasis by expression of an anti-idiotype in human commensal bacteria. Nat. Biotechnol. 18, 1060-1064 (2000).

46. Maassen, C.B. et al. Instruments for oral disease-intervention strategies: recombinant Lactobacillus casei expressing tetanus toxin fragment $C$ for vaccination or myelin proteins for oral tolerance induction in multiple sclerosis. Vaccine 17, 2117-2128 (1999)

47. Bleiweis, A.S., Lee, S.F., Brady, L.J., Progulske-Fox, A. \& Crowley, P.J. Cloning and inactivation of the gene responsible for a major surface antigen on Streptococcus mutans. Arch. Oral Biol. 35, 15S-23S (1990).

48. Bowen, W.H., Pearson, S.K. \& Young, D.A. The effect of desalivation on coronal and root surface caries in rats. J. Dent. Res. 67, 21-23 (1988).

49. Keyes, P.H. Dental caries in the molar teeth of rats. II. A method for diagnosing and scoring several types of lesions simultaneously. J. Dent. Res. 37, 1088-1099 (1958). 